\title{
Ultrasound-targeted microbubble destruction combined with dual targeting of HSP72 and HSC70 inhibits HSP90 function and induces extensive tumor-specific apoptosis
}

\author{
HANGHUI WANG $^{1 *}$, YIXIN SONG ${ }^{1 *}$, DINGJUN HAO ${ }^{2}$, MIN BAI $^{1}$, LIFANG JIN $^{1}$, JIYING GU ${ }^{1}$, \\ YIJIN SU ${ }^{1}$, LONG LIU ${ }^{1}$, CHAO JIA ${ }^{1}$ and LIANFANG DU ${ }^{1}$
}

${ }^{1}$ Department of Ultrasound, Shanghai First People's Hospital, School of Medicine, Shanghai Jiaotong University, Shanghai 200080; ${ }^{2}$ Hong Hui Hospital, Xi'an Jiaotong University College of Medicine, Shaanxi 710054, P.R. China

Received January 28, 2014; Accepted March 21, 2014

DOI: $10.3892 /$ ijo.2014.2388

\begin{abstract}
The specific and efficient delivery of small interfering RNA (siRNA) into cancer cells in vivo remains a major obstacle. In this study, we investigated whether ultrasoundtargeted microbubble destruction (UTMD) combined with dual targeting of HSP72 and HSC70 in prostate cancer cell lines improve the specific and efficient cell uptake of siRNA, inhibit HSP90 function and induce extensive tumor-specific apoptosis. VCaP cells were transfected with siRNA oligonucleotides. Cell viability assays were used to evaluate the safety of UTMD. The expression of HSP70, HSP90, caspase-8, caspase-3, PARP-1 and cleaved caspase-3 were determined by quantitative PCR and western blotting. Apoptosis and transfection efficiency were detected by flow cytometry. We found that HSP72, HSC70 and HSP90 expression was absent or weak in normal prostate epithelial cells (RWPE-1), and became uniformly and strongly expressed in prostate cancer cells (VCaP). VCaP and RWPE-1 cells expressed very low levels of caspase-8, caspase-3, PARP-1 and cleaved caspase-3. UTMD combined with dual targeting of HSP72 and HSC70 siRNA impoved the efficiency of transfection, cell uptake of siRNA, downregulated HSP70 and HSP90 expression in $\mathrm{VCaP}$ cells on the mRNA and protein levels, and upregulated major apoptotic markers (PARP-1, caspase-8, caspase-3 and cleaved caspase-3), thus, inducing extensive tumor-specific apoptosis. The Cell Counting Kit- 8 assay showed decreased
\end{abstract}

Correspondence to: Professor Lianfang Du, Department of Ultrasound, Shanghai First People's Hospital, School of Medicine, Shanghai Jiaotong University, 100 Hai Ning Road, Shanghai 200080, P.R. China

E-mail: lianfang_du@126.com

*Contributed equally

Abbreviations: UTMD, ultrasound-targeted microbubble destruction; siRNA, small interfering RNA; HRPC, hormone refractory prostate cancer; RT-PCR, reverse transcriptase-polymerase chain reaction

Key words: ultrasound-targeted microbubble destruction, small interfering RNA, hormone refractory prostate cancer, targeted therapy cellular viability in the HSP72/HSC70-siRNA silenced group. These results suggest that the combination of UTMD with dual targeting of HSP72 and HSC70 may improve the specific and efficient cell uptake of siRNA, inhibit HSP90 function and induce extensive tumor-specific apoptosis, indicating a novel, potential means for targeting therapeutic strategy to prostate cancer cells.

\section{Introduction}

Prostate cancer ( $\mathrm{PCa}$ ) is the most frequent malignancy and a leading cause of cancer-related deaths in American men, accounting for an estimated 233,000 (27\%) new cases and $29,480(10 \%)$ deaths in 2014 (1-3). Androgen deprivation therapy (ADT) provides an effective therapy for patients with advanced PCa. Although most patients with advanced PCa show an initial response to ADT, a significant percentage of patients invariably progress to hormone refractory prostate cancer (HRPC) and castration-resistant prostate cancer (CRPC) $(4,5)$. Effective targeting treatment for advanced CRPC and HRPC remains a critical clinical challenge. Thus, there is a clear need for novel targeted therapeutic strategies for the treatment of advanced PCa. siRNAs have provided us with clues how to tackle this issue. The properties of siRNA that are attractive for therapeutics include: i) stringent target-gene specificity, ii) relatively low immunogenicity of siRNA and iii) simplicity of design and testing of siRNA (6).

The HSP70 family includes at least eight members with diverse biochemical functions, including nascent protein folding, preventing denatured protein aggregation, and modulating assembly/disassembly of protein complexes $(7,8)$. The exact role of the HSP70 family in cancer remains to be elucidated. Firstly, the two major cytoplasmic isoforms are HSC70 and HSP72. Generally, HSC70 is abundantly and ubiquitously expressed in nontumor tissues, whereas HSP72 is present at relatively low levels in the absence of stress. Secondly, HSC70 and HSP72 expression may reduce sensitivity to HSP90 inhibitors. HSP70 as a survival tool for cancer cells. Targets of HSP70 could be upstream and downstream of mitochondria. However, targeting HSP70 or androgen receptor alone can result in off-target effets, drug resistance and disease recurrence. Combinatorial modula- 
tion of these two HSP70 isoforms could therefore be doubly advantageous (9-15).

However, the specific and efficient delivery of siRNA into cancer cells in vivo remains a major obstacle (16). The scientific researchers try to develop a technique that employs UTMD to deliver genes to specific cells or tissues in a multimodel approach. Microbubble contrast agents are emerging nonviral vector systems capable of binding nucleic acids, and that may overcome some of the limitations of current siRNA delivery systems. The use of microbubbles as nucleic acid delivery vectors is based on the observation that the rupture of microbubbles via UTMD results in deposition of microbubble shell components and increased cell membrane permeability localized to the site of microbubble-ultrasound interaction. This phenomenon has been exploited to deliver drug and genes both in vitro and in vivo. We and others have recently shown that UTMD can deliver therapeutic genes to tumors (17-21).

In the present study, we sought to use UTMD to achieve RNAi as a strategy prostate cancer cells growth suppression. Specifically, we tested the hypothesis that UTMD-mediated delivery of dual targeting of HSC70 and HSP72 siRNA will inhibits HSP90 function, induce extensive tumor-specifc apoptosis.

\section{Materials and methods}

Cell and cell culture. The human prostate carcinoma cell line VCaP and prostate epithelial cell RWPE-1 cell line were obtained from American Type Culture Collection (ATCC, Manassas, VA) and cultured accordingly. VCaP cell lines were cultured in DMEM (Invitrogen) supplemented with $10 \%$ fetal bovine serum (Gibco), $100 \mathrm{U} / \mathrm{ml}$ penicillin and $100 \mathrm{U} / \mathrm{ml}$ streptomycin (Invitrogen) and maintained in a humidified atmosphere of $5 \% \mathrm{CO}_{2}, 95 \%$ air at $37^{\circ} \mathrm{C}$. RWPE-1 cells were grown in Keratinocyte serum-free medium (K-SFM) medium (Invitrogen) supplemented with $5 \mathrm{ng} / \mathrm{ml}$ human recombinant EGF and $0.05 \mathrm{mg} / \mathrm{ml}$ bovine pituitary extract under standard cell culture conditions.

Experimental groups. VCaP cells were divided into the following 8 groups for gene transfection experiment. The control group (NC); HSP72 siRNA, HSC70-siRNA, HSP72/ HSC70-siRNA; UTMD+HSP72-siRNA, UTMD+HSC70siRNA, UTMD+HSP72/HSC70-siRNA; UTMD.

UTMD exposure protocols. A therapeutic ultrasound machine (Physioson-Basic, Physioson Elektromedizin, Germany) was used to emit ultrasound at the frequency of $1 \mathrm{MHz}$. The area of the ultrasound probe was $2.5 \mathrm{~cm}^{2}$. The ultrasound transducer was placed at the bottom of plates or dishes with coupling medium on the surface of the transducer. The adjustable sonication parameters included ultrasound intensity, exposure time, pulse frequency, and duty cycle.

Microbubbles (SonoVue, Bracco, Milan, Italy) were lipid-shelled ultrasound contrast agents containing sulfur hexafluoride gas (diameter 2.5-6.0 $\mu \mathrm{m}$ ) and used at a concentration of $\sim 2 \times 10^{8}$ bubbles $/ \mathrm{ml}$. The volumetric ratio of microbubbles to medium dictated the choice of contrast agent dose.

After protocol optimization with several various settings, the following UTMD parameters were used: ultrasound inten- sity, $1 \mathrm{~W} / \mathrm{cm}^{2}$; exposure time, $60 \mathrm{sec}$; pulse frequency, $100 \mathrm{~Hz}$; duty cycle, $20 \%$; volumetric ratio of microbubbles: medium, 1:5.

siRNA-mediated gene knockdown. siRNAs (Shanghai GenePharma Co., Ltd.) were designed against the open reading frame of HSP72 (HSPA1A; accession number NM_005345) or HSC70 (HSPA8; accession number NM_006597). Two active sequences were used for studies against HSP72 or HSC70 (designated HSP72 or HSC70). Active sequences for HSP72 and HSC70 are as follows: HSP72 (sense: GGACGAGUUUG AGCACAAGTT, antisense: CUUGUGCUCAAACUCGUC CTT), HSC70 (sense: CCAAGCAGACGCAGAUCUUTT, antisense: AAGAUCUGCGUCUGCUUGGTT), Negative control (sense: UUCUCCGAACGUGUCACGUTT, antisense: ACGUGACACGUUCGGAGAATT). For all experiments cells were transfected with siRNA (200 nM for single transfections or $100 \mathrm{nM}$ for combinatorial transfections) in Opti-MEM ${ }^{\circledR}$ medium without serum according to the Lipofectamine ${ }^{\circledR} 2000$ protocol (Invitrogen). An equal amount of scrambled Stealth siRNA was used as a negative control. Six hours after the cells were transfected, the medium was replaced with fresh culture medium. All experiments were performed $48 \mathrm{~h}$ after transfection and repeated three times.

Gene transfection efficiency. Following gene transfection, the effciency of transfection was evaluated by fuorescence microscopy and flow cytometry. Green fuorescence was detected using inverted fuorescence microscopy (Zeiss Axiovert S100; Carl Zeiss, Jena, Germany). The percentage of FAM expression of the transducted $\mathrm{VCaP}$ cells was quantitatively measured by flow cytometry (FACSCalibur; BD Biosciences, Franklin Lakes, NJ, USA).

Cell viability assays. A Cell Counting Kit-8 assay (CCK-8, Dojindo Laboratories) was used to evaluate relative cell viability. At $48 \mathrm{~h}$ after siRNA transfections, RWPE-1 and $\mathrm{VCaP}$ cells were plated in 96 -well microtiter plates at a density of $5 \times 10^{3}$ cells per well, then the live cell count was assayed using CCK- 8 according to the manufacturer's instructions. Briefly, $10 \mu \mathrm{l}$ of CCK8 solution was added to each well, and the absorbance at $450 \mathrm{~nm}$ was measured using a Thermomax microplate reader (Molecular Devices, Hercules, CA, USA) after 1-4 h of incubation. Relative cell viability was calculated as a percentage of untreated control cells.

Flow cytometry. Cells were trypsinized and resuspended in Opti-MEM medium and plated on 6-well plates at 1,000 cells/well after transfection with siRNA for $48 \mathrm{~h}$. For apoptosis detection, cells were stained with Annexin $\mathrm{V}$ and propidium iodide using the Annexin V-FITC Apoptosis Detection kit (Invitrogen), and the percentage of apoptotic cells was determined by flow cytometry (Beckman Coulter).

Quantitative real-time PCR. Total RNA from cultured cells was extracted using TRIzol reagent (Invitrogen) and reverse transcription was performed by PrimeScript ${ }^{\circledR}$ RT Master mix Perfect real-time (Takara) according to the manufacturer's instructions. RNA integrity was examined by agarose gel electrophoresis. Quantitative RT-PCR was performed in triplicate using the SYBR-Green (Takara) method on an Applied 

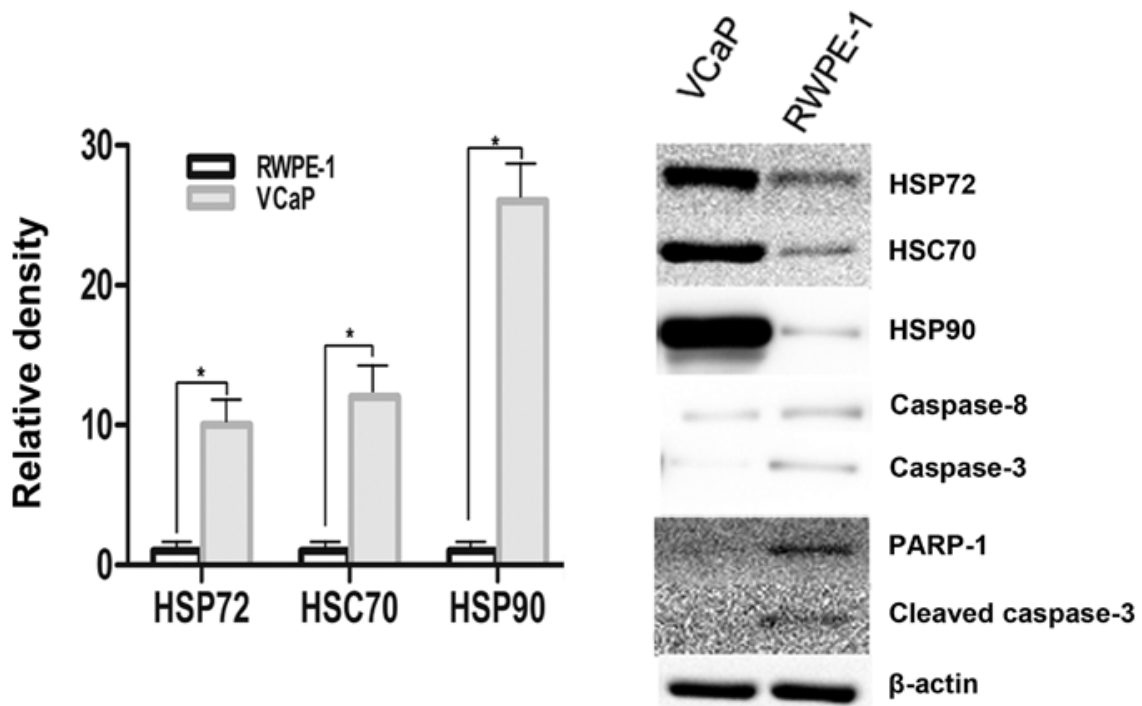

Figure 1. HSP72, HSC70 and HSP90 is overexpressed in VCaP compared with RWPE-1. Representative images showing expression of HSP72, HSC70 and HSP90 protein in VCaP and RWPE-1 cells as analyzed by western blotting $(\mathrm{P}<0.05)$. Relative density of HSP72, HSC70 and HSP90 are shown in the left panel. All experiments were repeated three times with similar results. The main hallmark of apoptosis (cleaved caspase-8, cleaved caspase-3, cleaved PARP-1, caspase-3) were also detected.

Biosystems Fast Sequence Detector System. Conditions for each target were validated by standard and melting curve analyses. We used $\beta$-actin expression as an internal control. Expression was normalized to $\beta$-actin and determined using the Applied Biosystems 7500. All primers were designed using Primer 5 and synthesized by Shanghai Sunny Biotechnology Co., Ltd. The following primers were used: HSP72 (forward, 5'-TGGAGTCCTACGCCTTCAAC-3'; reverse, 5'-AGCCAC GAGATGACCTCTTG-3'), HSC70 (forward, 5'-CGCAAGC ATAAGAAGGACATCA-3'; reverse, 5'-GAACAGGTCAGC ATTCAGTTCT-3'), $\beta$-actin (forward, 5'-GGAGATTACTGC CCTGGCTCCTA-3'; reverse, 5'-GACTCATCGTACTCCT GCTTGCTG-3'). Caspase-3 (forward, 5'-GAACTGGACTG TGGCATTGAGA-3'; reverse, 5'-ATGGCACAAAGCGAC TGGATGA-3'). HSP90 (forward, 5'-TCTTGGCACCACCT ACTCTTGT-3'; reverse, 5'-CATCACCGATCAACCGT TCAGT-3').

Western blot analysis. Prostate cells transfected with siRNA were washed twice in ice-cold phosphate buffer solution (PBS), harvested and then lysed using RIPA buffer (Sigma). Protein concentrations were quantified using the BCA Protein assay kit (Thermo Scientific, Rockford, IL, USA). Equal amounts of protein and PageRuler ${ }^{\mathrm{TM}}$ Prestained molecular weight markers (Thermo Scientific) were separated by $8 \%$ or $10 \%$ SDS-PAGE and transferred to $0.45 \mu \mathrm{m}$ pore size PVDF membrane (Millipore). Membranes were blocked in TBS with Tween-20 containing 5\% skim milk (Bright Dairy) and incubated with the primary antibody at room temperature for $1 \mathrm{~h}$ in blocking buffer. Antibodies and dilutions were 1:2,000 for anti-HSP72 (C92F3A-5, Stressgen Biotech Corp.), 1:1,000 for anti-caspase-8 (1006-1, Epitomics), 1:2,000 for antiHSP90 (2877-1, Epitomics), 1:50,000 for anti-PARP-1 (1051-1, Epitomics), 1:5,000 for anti-HSC70, anti-caspase-3 (1776-1, 1087-1,Epitomics), 1:2,000 for anti-cleaved caspase-3, and anti$\beta$-actin $(9664,4970$, Cell Signaling Technology) antibodies. Washing steps after hybridization were three times at room
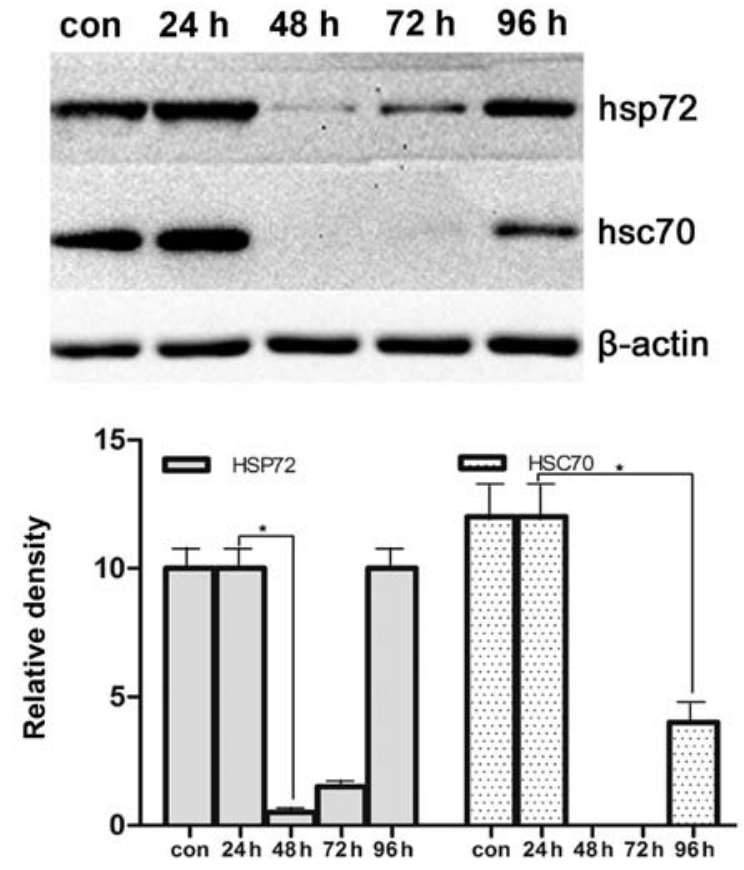

Figure 2. Optimal time points of efficacy of silencing HSP70 gene by HSP72-siRNA transfected. The best time points of efficacy of silencing HSP70 gene was at $48 \mathrm{~h}(\mathrm{P}<0.05)$. Relative density of HSP72 and HSC70 are shown in the lower panel. VCaP and RWPE-1 cells expressed very low levels of caspase-8, caspase-3, PARP-1 and cleaved caspase-3.

temperature for $10 \mathrm{~min}$ for all other antibodies. Membranes were incubated with secondary HRP-conjugated antibodies diluted 1:2,000 in blocking buffer and at room temperature for $1 \mathrm{~h}$. After three washing steps of $10 \mathrm{~min}$ at room temperature, detection of the signals was performed using the Immobilon Western Chemiluminescent HRP Substrate (Millipore).

Statistical analysis. Statistical analysis was carried out with SPSS version 12.0 (SPSS, Inc., Chicago, IL, USA). All data are 

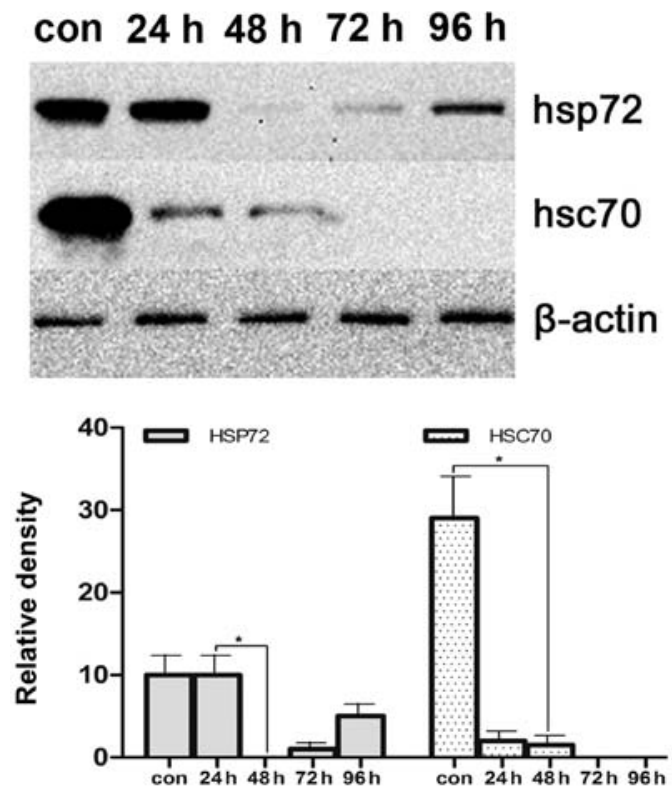

Figure 3. Optimal time points of efficacy of silencing HSP70 gene by HSC70-siRNA transfection. The best time points of efficacy of silencing HSP70 gene was at $48 \mathrm{~h}(\mathrm{P}<0.05)$. Relative density of HSP72 and HSC70 are shown in the lower panel.

presented as mean \pm SD and analyzed by One-way ANOVA. Values were considered statistically significant at $\mathrm{P}<0.05$. Results are representative of more than three individual experiments.

\section{Results}

HSP72, HSC70 and HSP90 is overexpressed in human prostate cancer cells compared with human prostate epithelial cells. HSP70 is a critical co-chaperone for HSP90. HSP70 and
HSP90 are important therapeutic biomarker of prostate cancer. Caspase- 8 is a member of a family of cysteine-requiring aspartate proteases that participate in the intracellular signaling cascade leading to apoptosis (programmed cell death) (22). Poly (ADP-ribose) polymerase (PARP) is zinc-dependent DNA binding protein that recognizes DNA strand breaks and is presumed to play a role in DNA repair. As a marker for apoptosis, PARP is cleaved in vitro by many caspases (23). We detected the cleaved caspase- 8 and p25 cleaved-form of PARP-1 (Cleaved p25). We compared gene expression profiles of VCaP and RWPE-1 in vitro, and found that HSP72, HSC70, and HSP90 was overexpressed in VCaP cells. Western blot analysis revealed that $\mathrm{VCaP}$ cells expressed higher levels of HSP72, HSC70 and HSP90 protein in vitro compared with RWPE-1, which expressed undetectable levels. VCaP and RWPE-1 cells expressed very low levels of caspase-8, caspase-3, PARP-1 and cleaved caspase-3 (Fig. 1).

Effects of HSP70 expression after transfection at different time points. To confirm the optimal time points in HSP70 expression in vitro after transfection, we selected $24,48,72,96 \mathrm{~h}$ to quantify the expression of HSP70 protein in VCaP cells. As shown in Figs. 2 and 3, optimal time points of efficacy of silencing HSP70 gene by siRNA is $48 \mathrm{~h}$. Therefore, this was used for further investigation after transfection in vitro.

Gene transfection efficiency and apoptosis. Fig. 4 shows the UTMD+HSC70/HSP72-siRNA group had maximal transfection percentage $(73.15 \pm 0.53)$ and it was significantly higher than HSC70/HSP72-siRNA group (40.37 \pm 0.65$)$, UTMD+HSC70siRNA group $(28.31 \pm 0.60)$ and UTMD+HSP72-siRNA group (24.25 \pm 0.53 ). In accordance with the results of transfection analysis, the apoptosis percentage of the UTMD+HSC70/ HSP72-siRNA group was the highest $(47.16 \%)$ and the other 6 groups had significant difference between each other $(\mathrm{P}<0.05)$.
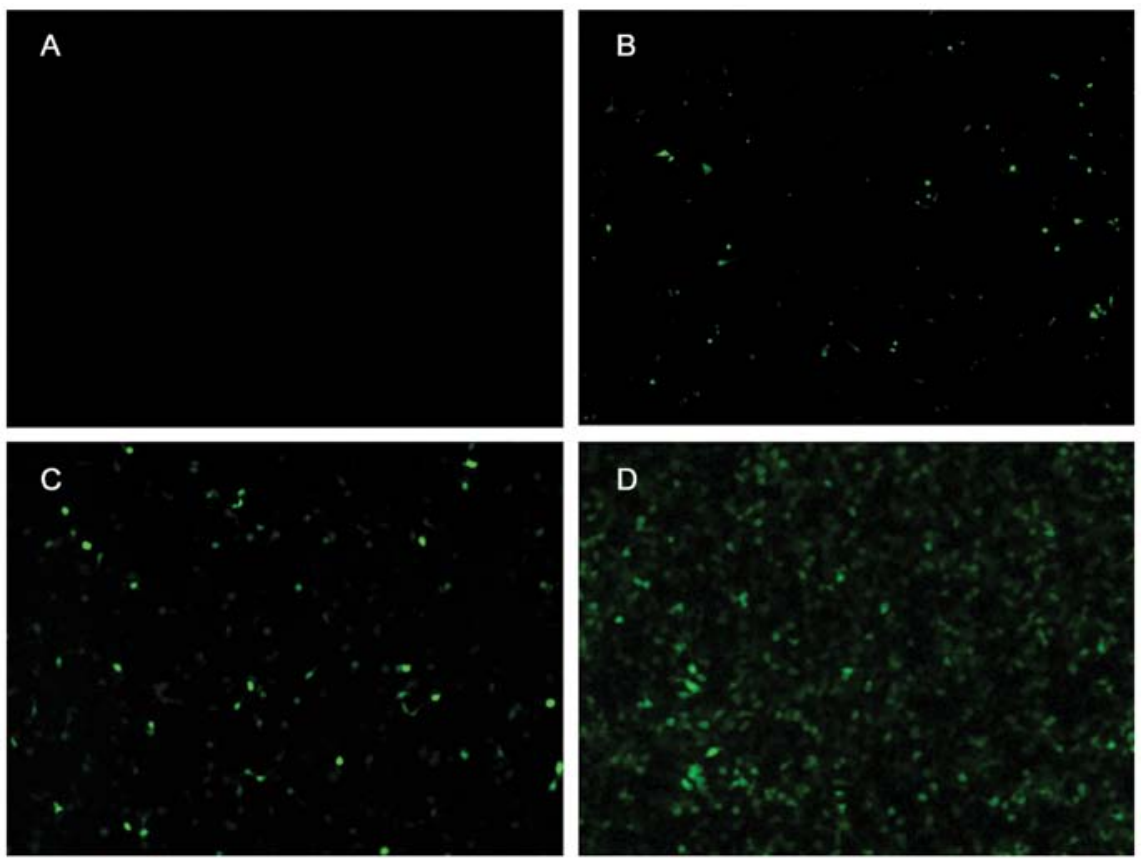

Figure 4. The transfection percentage of UTMD+HSC70/HSP72-siRNA group improved (P<0.05). (A) Control, (B) HSC70-siRNA, (C) HSP72/HSC70 siRNA, (D) HSP72/HSC70 siRNA+UTMD. 


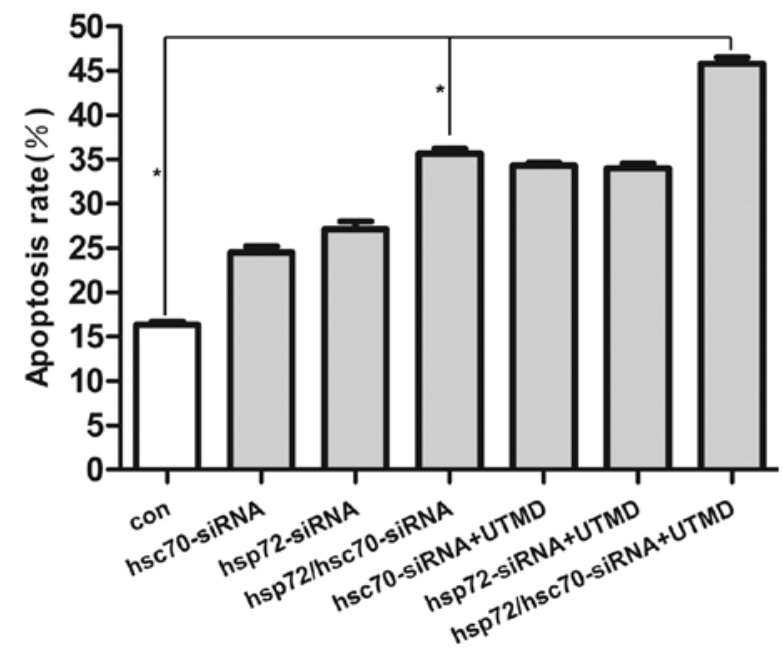

Figure 5. Apoptosis assay of VCaP cells transfected with HSP70 siRNA. Apoptosis rates of 3 groups are shown, compared with siRNA group and UTMD plus siRNA group $(\mathrm{P}<0.01)$.

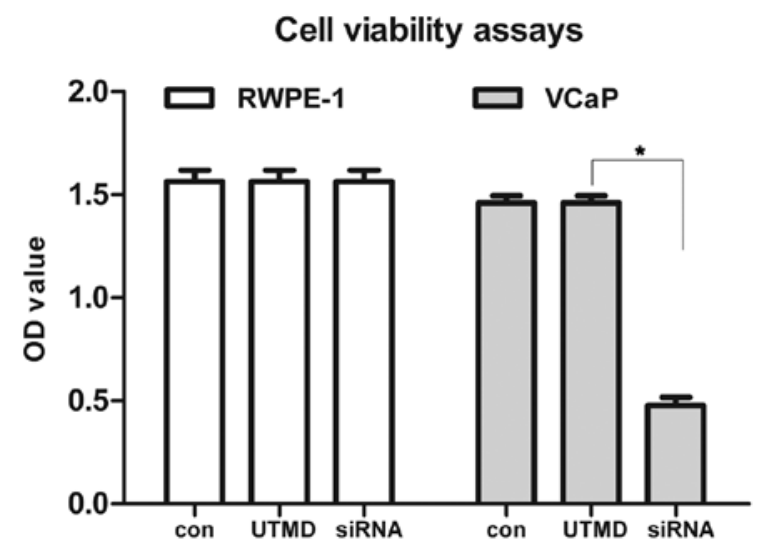

Figure 6. Viability of RWPE-1 and VCaP cells in the presence of UTMD and siRNA. The cellular viability of VCaP cells transfected with HSP72 and HSC70 siRNA was delayed compared with that of the control groups after UTMD ( $\mathrm{P}<0.01)$. UTMD is a safety and noninvasive approach for RWPE-1.

It seems that the UTMD+HSC70/HSP72-siRNA could serve as a gene delivery system, and silencing HSP70 and HSP90 expression induced extensive cell apoptosis (Fig. 5).

UTMD is a safe and noninvasive approach. In order to investigate the safety of UTMD, cellular viability was monitored using the Cell Counting Kit-8 assay for $48 \mathrm{~h}$. The cellular viability of VCaP cells transfected with HSP72 and HSC70 siRNA was delayed compared with that of the control groups after UTMD. No significant difference was found in the cellular viability between the RWPE- 1 and $\mathrm{VCaP}$ of the control group and the two group treated with UTMD (Fig. 6).

To investigate the molecular mechanisms by which the combination of UTMD and HSP70-siRNA restrains PCa in the context of HRPC, we processed a set of relevant experiment in vitro. RT-qPCR revealed 3.1-fold higher levels of HSP72, HSC70 and HSP90 mRNA in NC control compared with HSP72/HSC70-siRNA treatment (Figs. 7-10). The group of UTMD combined with HSP72/HSC70-siRNA expressed undetectable levels of HSP72, HSC70 and HSP90 mRNA. This

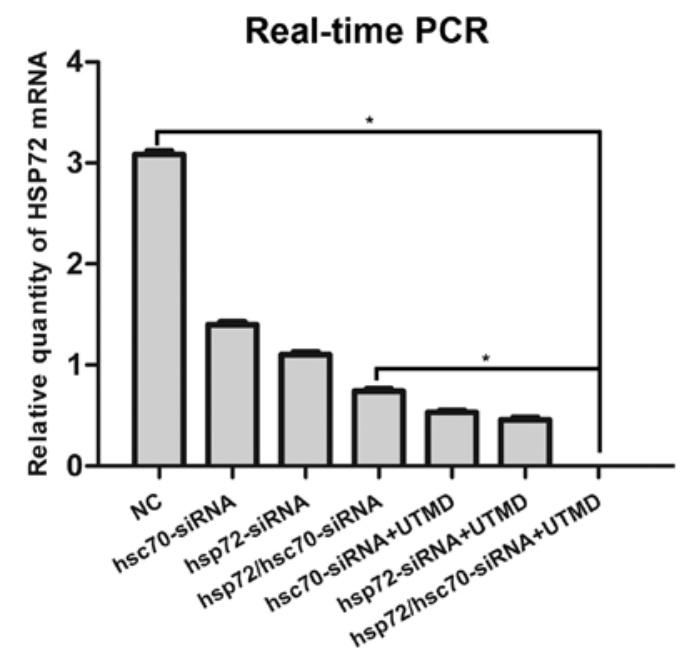

Figure 7. Real-time qPCR analysis of mRNA HSP72 expression in VCaP cells. UTMD combination with dual targeting HSP72 and HSC70 siRNA had better gene silencing efficiency of HSP72 than the other groups $(\mathrm{P}<0.01)$.

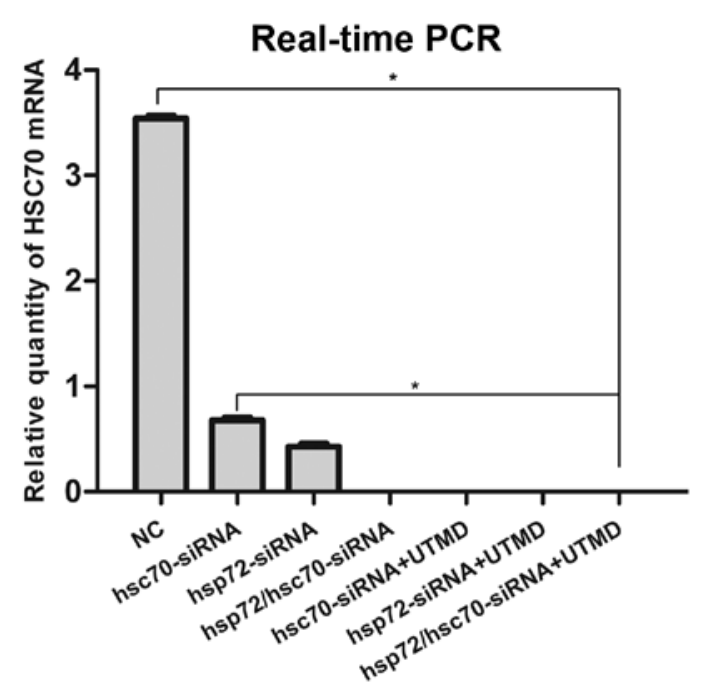

Figure 8. Real-time qPCR analysis of mRNA HSC70 expression in VCaP cells. UTMD combination with dual targeting HSP72 and HSC70 siRNA had better gene silencing efficiency of HSC70 than the other groups $(\mathrm{P}<0.01)$.

is consistent with the results of western blotting in $\mathrm{VCaP}$ cells (Figs. 11 and 12). To better understand the biological processes that underlie what appears to be HSP70, HSP90, and cleaved caspase-3 in VCaP cells, we next sought to define the mechanism by which HSP70 regulates cleaved caspase-3 expression. We found that the caspase-3, caspase-8, PARP-1, and cleaved caspase- 3 gene was the most significantly enriched in the group of UTMD combined HSP72/HSC70-siRNA (Figs. 10 and 11). Therefore, we tested the ability of the combination of UTMD with HSP72/HSC70-siRNA to induce tumor specific apoptosis.

\section{Discussion}

Silencing of gene expression by siRNA is rapidly becoming a powerful tool against cancer, efficient delivery of siRNA into tumor cells remains a key obstacle (24). Although local surgery, radiation, or hormonal ablation provide initial response at early 


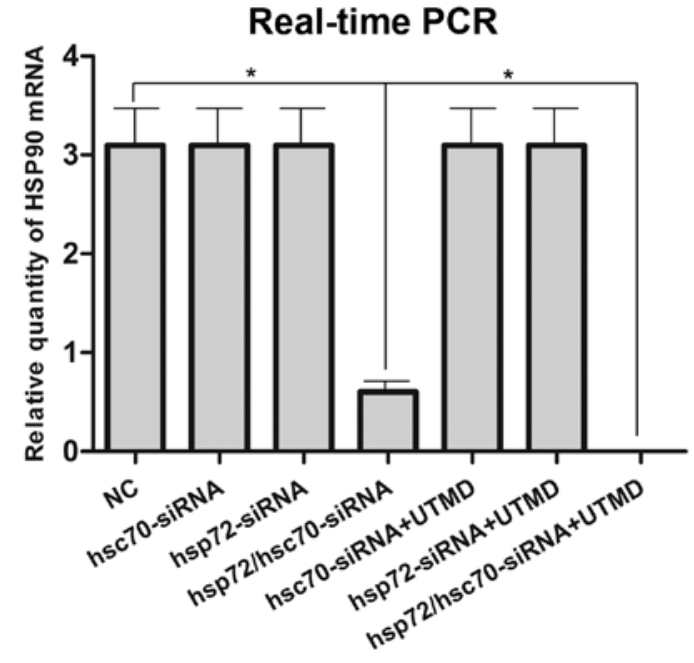

Figure 9. Real-time qPCR analysis of mRNA HSP90 expression in $\mathrm{VCaP}$ cells. UTMD combination with dual targeting HSP72 and HSC70 siRNA had better gene silencing efficiency of HSP90 than the other groups $(\mathrm{P}<0.01)$

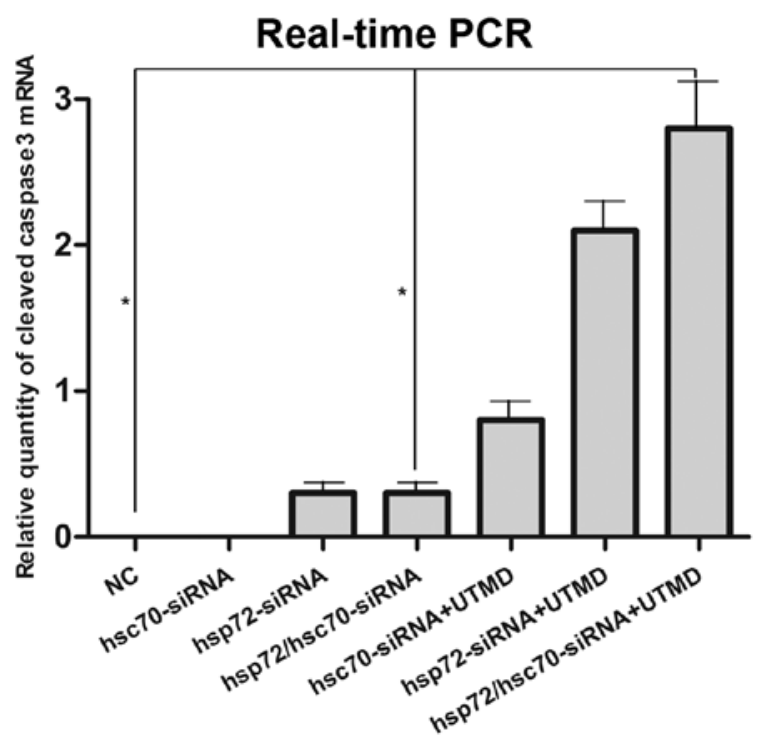

Figure 10. Real-time qPCR analysis of mRNA cleaved caspase-3 expression in VCaP cells. UTMD combination with dual targeting HSP72 and HSC70 siRNA had better gene expression of cleaved caspase- 3 than the other groups $(\mathrm{P}<0.01)$.

stages of the disease, tumor cells often develop resistance and relapse. Thus, the identification of new therapeutic targets for prostate cancer is of critical importance. Recent studies further revealed that $\approx 2 / 3$ of human prostate cancers expressed high level of HSP72 and HSC70, associating with recurrence, development of castration-resistance, and poor survival $(25,26)$. These studies provide the first hints that HSP72 and HSC70 may have a critical role in prostate cancer development and therapeutic resistance. HSP90 is an exciting therapeutic target in cancer because inhibition of this single protein causes the simultaneous degradation of multiple oncoproteins and combinatorial blockade of numerous oncogenic pathways. HSP70 molecular chaperones are of interest when considering modulation of HSP90 (27). HSP70 is the major therapeutic target in advanced PCa (28-32). However, targeting HSP70 or androgen

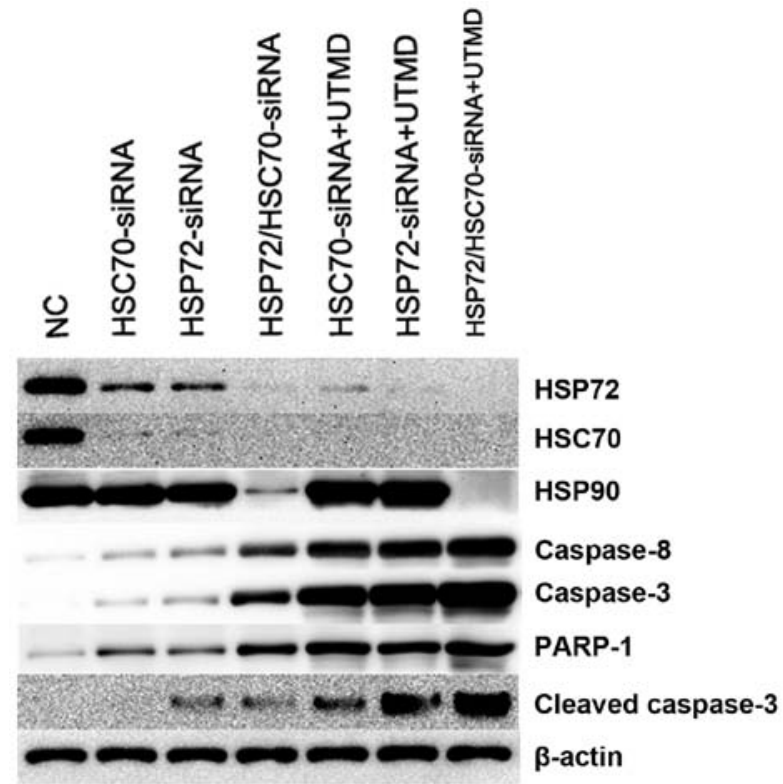

Figure 11. Western blot analysis of expression of HSP72, HSC70, HSP90, caspase-8, caspase-3, PARP-1 and cleaved caspase-3 protein in VCaP cells at $48 \mathrm{~h}$ after transfection. One of three independent experiments is shown. The caspase- 8 and PARP-1 are cleaved.

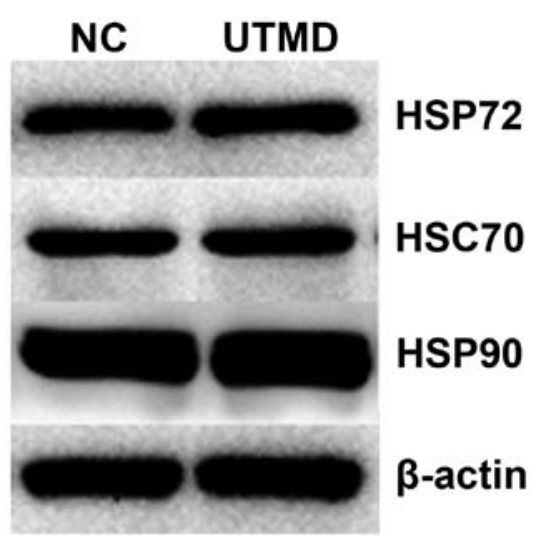

Figure 12. Western blot result of the UTMD group. No change in the expression of HSP72, HSC70, HSP90 between the UTMD group and control group.

receptor alone can result in off-target effects, drug resistance and disease recurrence $(30,33,34)$. Therefore, simultaneous targeting of a multi-modal approach or multiple pathways in targeted delivery could in principle be an effective approach to treating prostate cancer. With respect to the functional role of HSP70 isoforms in the HSP90 chaperone, the data we presented here demonstrate that silencing HSC70 or HSP72 individually has little effect on HSC70 and HSP72, has no effect on HSP90 protein level of $\mathrm{VCaP}$ prostate cancer cells, while dual targeting of HSC70 and HSP72 inhibit the cellular chaperone activity of HSP90, and has good silencing effect on HSC70 and HSP72, thus induce tumor-specifc apoptosis. Additionally, UTMD combined with simultaneous targeting of HSC70 and HSP72 may further enhance silencing efficacy of the expression of HSP72, HSC70, HSP90 protein and mRNA. In agreement with our findings, Powers et al (34) also found that dual targeting of HSC70 and HSP72 silence HSP90 protein and induce extensive 
tumor-specifc apoptosis in human HCT116 colon and A2780 ovarian cells.

In the present study, we have shown by flow cytometry that silencing HSC70 or HSP72 individually has no effect on apoptosis. Using combinatorial siRNA approach, we revealed that dual silencing of HSC70 and HSP72 considerably increased the apoptotic efficacy by $20 \%$ compare with control group, UTMD may further enhance extensive tumorspecific apoptosis by $11 \%$ (Fig. 5). Our apoptosis results were in accord with the study of Rérole et al (35). In order to confirm further the results of flow cytometry, we selected several major apoptotic markers. Caspase-3, caspase-8, PARP-1 and cleaved caspase- 3 are the main executor of apoptosis and considered to be key hallmarks of apoptosis $(36,37)$. Our data on apoptosis is in near agreement with the expression of major apoptotic markers (caspase-8, caspase-3, PARP-1, cleaved caspase-3) of western blotting. Of note, the safety and noninvasiveness of the combinational targeting strengthen our confidence further. UTMD is a noninvasive physical gene delivery technology by sonoporation. There is no significant difference of cell viability in the UTMD group and normal control group, indicating that UTMD cells without any apparent toxicity, but also UTMD can promote effective uptake of siRNA, and enhance the specificity of siRNA to silence HSP70 and HSP90. Therefore, we may promote the efficacy and specificity of siRNA delivered by UTMD. UTMD may improve the accuracy of target genes. Moreover, these data indicate that this is a promising strategy for inducing tumor-specific apoptosis in vitro. The safety of the combinational targeting in vivo need to be explored in subsequent experiments.

The most important attribute of UTMD combination with siRNA is their specific, efficient delivery, which leads to concentration in the target tumor and avoidance of distribution to irrelevant normal tissues. Specific delivery into the intended target cells will likely reduce the dose required for antitumor activity as well as limit toxicity.

An exciting implication of our research is the attractiveness of combinatorial targeting of HSC70 and HSP72 as an alternative means to achieve HSP90 inhibition, with the added advantage of avoiding the anti-apoptotic effects of HSP70 isoform induction that limit the use of current pharmacologic inhibitors. Noteworthy, UTMD may promote the effect of silencing HSP90. The approach of UTMD combined siRNA was used here as a tool to silence the expression of HSC70, HSP72 and HSP90. It is possible that UTMD combined with RNA interference may be developed as a potential therapeutic approach.

Our findings also revealed further details about the relationship between UTMD and siRNA, especially in the context of HRPC. The reasons why UTMD combined with dual targeting of HSC70 and HSP72 improve the efficacy of silencing are: Firstly, UTMD promote the efficient delivery and cell uptake of siRNA. Secondly, UTMD also may activate the active region of HSP72, HSC70, HSP90 or increase crosstalk between distinct signaling pathways. Thirdly, UTMD combined with dual targeting of siRNA might active multiple signal transduction pathways of apoptosis and heat shock protein. Fourthly, UTMD improve the permeability and ATP energy of mitochondria. Further work is required to identify the molecular and cellular mechanisms whereby UTMD promote dual targeting of siRNA induce extensive tumor-specifc apoptosis in vitro and in vivo from the results presented here.

In conclusion, UTMD-mediated delivery of dual targeting of siRNA is a promising strategy for treatment of prostate cancer in vitro that could prove more effective and specific than other siRNA delivery strategies. The other main finding of this study is the potential superiority for HSP70 and HSP90 as biomarker targeting in PCa $(33,38)$. Our data establish that UTMD-directed simultaneous targeting of siRNA therapeutics is minimally invasive, and effective at suppressing tumor growth, and induce extensive tumor-specifc apoptosis, and these data might provide insights into further optimization and the multi-model delivery strategy is versatile in specific gene silencing of targeted therapeutics of cancer.

\section{Acknowledgements}

We thank members of the Guo Hui Fu Laboratory for helpful discussions. We thank Dr J.J. Yu for technical assistance. This study was supported by the National Natural Science Foundation of China; grant no. 81171352 and 81271596 (to L. Du), no. 81201097 (to M. Bai), and the Natural Science Foundation of Shanghai no. 12ZR1424800 (to M. Bai) is gratefully acknowledged.

\section{References}

1. Siegel R, Ma J, Zou Z, et al: Cancer statistics, 2014. CA Cancer J Clin 64: 9-29, 2014.

2. DeSantis C, Naishadham D and Jemal A: Cancer statistics for African Americans, 2013. CA Cancer J Clin 63: 151-166, 2013.

3. Jemal A, Bray F, Center MM, et al: Global cancer statistics. CA Cancer J Clin 61: 69-90, 2011.

4. Bitting RL and Armstrong AJ: Targeting the PI3K/Akt/mTOR pathway in castration-resistant prostate cancer. Endocr Relat Cancer 20: R83-R99, 2013.

5. Garcia JA and Rini BI: Castration-resistant prostate cancer: Many treatments, many options, many challenges ahead. Cancer 118: 2583-2593, 2012.

6. McNamara JO II, Andrechek ER, Wang Y, et al: Cell typespecific delivery of siRNAs with aptamer-siRNA chimeras. Nat Biotechnol 24: 1005-1015, 2006.

7. Daugaard $M$, Rohde $M$ and Jäättelä $M$ : The heat shock protein 70 family: highly homologous proteins with overlapping and distinct functions. FEBS Lett 581: 3702-3710, 2007.

8. Zuiderweg ER, Bertelsen EB, Rousaki A, et al: Allostery in the Hsp70 chaperone proteins. Top Curr Chem 328: 99-153, 2013.

9. Davenport EL, Zeisig A, Aronson LI, et al: Targeting heat shock protein 72 enhances Hsp90 inhibitor-induced apoptosis in myeloma. Leukemia 24: 1804-1807, 2010.

10. Powers MV, Jones K, Barillari C, et al: Targeting HSP70: the second potentially druggable heat shock protein and molecular chaperone? Cell Cycle 9: 1542-1550, 2010.

11. Meng L, Hunt C, Yaglom JA, et al: Heat shock protein Hsp72 plays role in Her2-induced mammary tumorigenesis. Oncogene 30: 2836-2845, 2011.

12. Stangl S, Gehrmann M, Riegger J, et al: Targeting membrane heat-shock protein 70 (Hsp70) on tumors by cmHsp70.1 antibody. Proc Natl Acad Sci USA 108: 733-738, 2011.

13. Evans CG, Chang L and Gestwicki JE: Heat shock protein 70 (hsp70) as an emerging drug target. J Med Chem 53: 4585-4602, 2010.

14. Balaburski GM, Leu JI, Beeharry N, et al: A modified HSP70 inhibitor shows broad activity as an anticancer agent. Mol Cancer Res 11: 219-229, 2013.

15. Rérole AL, Gobbo J, De Thonel A, et al: Peptides and aptamers targeting HSP70: a novel approach for anticancer chemotherapy. Cancer Res 71: 484-495, 2011.

16. Yao YD, Sun TM, Huang SY, et al: Targeted delivery of PLK1-siRNA by ScFv suppresses HER $2^{+}$breast cancer growth and metastasis. Sci Transl Med 4: 130ra48, 2012. 
17. Li HL, Zheng XZ, Wang HP, et al: Ultrasound-targeted microbubble destruction enhances AAV-mediated gene transfection in human RPE cells in vitro and rat retina in vivo. Gene Ther 16 : 1146-1153, 2009.

18. Xie W, Liu S, Su H, et al: Ultrasound microbubbles enhance recombinant adeno-associated virus vector delivery to retinal ganglion cells in vivo. Acad Radiol 17: 1242-1248, 2010.

19. Zheng X, Du L, Wang H and Gu Q: A novel approach to attenuate proliferative vitreoretinopathy using ultrasound-targeted microbubble destruction and recombinant adeno-associated virus-mediated RNA interference targeting transforming growth factor- $\beta 2$ and platelet-derived growth factor-B. J Gene Med 14: 339-347, 2012.

20. Suzuki R, Oda Y, Utoguchi N and Maruyama K: Progress in the development of ultrasound-mediated gene delivery systems utilizing nano- and microbubbles. J Control Release 149: 36-41, 2011.

21. Tachibana K, Uchida T, Ogawa K, et al: Induction of cellmembrane porosity by ultrasound. Lancet 353: 1409, 1999.

22. Boldin MP, Goncharov TM, Goltsev YV and Wallach D: Involvement of MACH, a novel MORT1/FADD-interacting protease, in Fas/APO-1- and TNF receptor-induced cell death. Cell 85: 803-815, 1996.

23. Lazebnik YA, Kaufmann SH, Desnoyers S, et al: Cleavage of poly(ADP-ribose) polymerase by a proteinase with properties like ICE. Nature 371: 346-347, 1994.

24. Pai SI, Lin YY, Macaes B, et al: Prospects of RNA interference therapy for cancer. Gene Ther 13: 464-477, 2006.

25. Daneshmand S, Quek ML, Lin E, et al: Glucose-regulated protein GRP78 is up-regulated in prostate cancer and correlates with recurrence and survival. Hum Pathol 38: 1547-1552, 2007.

26. Pootrakul L, Datar RH, Shi SR, et al: Expression of stress response protein Grp78 is associated with the development of castrationresistant prostate cancer. Clin Cancer Res 12: 5987-5993, 2006.
27. Whitesell L and Lindquist SL: HSP90 and the chaperoning of cancer. Nat Rev Cancer 5: 761-772, 2005.

28. Lebret T, Watson RW and Fitzpatrick JM: Heat shock proteins: their role in urological tumors. J Urol 169: 338-346, 2003.

29. Garrido C, Schmitt E, Candé C, et al: HSP27 and HSP70: potentially oncogenic apoptosis inhibitors. Cell Cycle 2: 579-584, 2003.

30. McConnell JR and McAlpine SR: Heat shock proteins 27, 40, and 70 as combinational and dual therapeutic cancer targets. Bioorg Med Chem Lett 23: 1923-1928, 2013.

31. Alaiya AA, Oppermann M, Langridge J, et al: Identification of proteins in human prostate tumor material by two-dimensional gel electrophoresis and mass spectrometry. Cell Mol Life Sci 58: 307-311, 2001.

32. Mayer MP: Hsp70 chaperone dynamics and molecular mechanism. Trends Biochem Sci 38: 507-514, 2013.

33. Lee E, Madar A, David G, et al: Inhibition of androgen receptor and $\beta$-catenin activity in prostate cancer. Proc Natl Acad Sci USA 110: 15710-15715, 2013

34. Powers MV, Clarke PA and Workman P: Dual targeting of HSC70 and HSP72 inhibits HSP90 function and induces tumorspecific apoptosis. Cancer Cell 14: 250-262, 2008.

35. Rérole AL, Jego G and Garrido C: Hsp70: anti-apoptotic and tumorigenic protein. Methods Mol Biol 787: 205-230, 2011.

36. Earnshaw WC, Martins LM and Kaufmann SH: Mammalian caspases: structure, activation, substrates, and functions during apoptosis. Annu Rev Biochem 68: 383-424, 1999.

37. Tang NY, Huang YT, Yu CS, et al: Phenethyl isothiocyanate (PEITC) promotes G2/M phase arrest via p53 expression and induces apoptosis through caspase- and mitochondria-dependent signaling pathways in human prostate cancer DU 145 cells. Anticancer Res 31: 1691-1702, 2011.

38. Goloudina AR, Demidov ON and Garrido C: Inhibition of HSP70: a challenging anti-cancer strategy. Cancer Lett 325: 117-124, 2012. 